\title{
The MEKK1-JNK pathway plays a protective role in pressure overload but does not mediate cardiac hypertrophy
}

\author{
Junichi Sadoshima, ${ }^{1}$ Olivier Montagne, ${ }^{1}$ Qian Wang, ${ }^{1}$ Guiping Yang, ${ }^{1}$ Jill Warden, ${ }^{1}$ \\ Jing Liu, ${ }^{1}$ Gen Takagi, ${ }^{1}$ Vijaya Karoor, ${ }^{1}$ Chull Hong, ${ }^{1}$ Gary L. Johnson, ${ }^{2}$ \\ Dorothy E. Vatner, ${ }^{1}$ and Stephen F. Vatner ${ }^{1}$ \\ ${ }^{1}$ Cardiovascular Research Institute, Department of Cell Biology and Molecular Medicine, and Department of Medicine, \\ University of Medicine and Dentistry of New Jersey, New Jersey Medical School, Newark, New Jersey, USA \\ ${ }^{2}$ University of Colorado Cancer Center, University of Colorado Health Science Center School of Medicine, \\ Denver, Colorado, USA
}

\begin{abstract}
Mitogen-activated protein kinase kinase kinase (MEKK1) mediates activation of c-Jun $\mathrm{NH}_{2}$-terminal kinase (JNK). Although previous studies using cultured cardiac myocytes have suggested that the MEKK1-JNK pathway plays a key role in hypertrophy and apoptosis, its effects in cardiac hypertrophy and apoptosis are not fully understood in adult animals in vivo. We examined the role of the MEKK1-JNK pathway in pressure-overloaded hearts by using mice deficient in MEKK1. We found that transverse aortic banding significantly increased JNK activity in Mekk1 ${ }^{+/+}$but not Mekk1 $1^{-/-}$mice, indicating that MEKK1 mediates JNK activation by pressure overload. Nevertheless, pressure overload caused significant levels of cardiac hypertrophy and expression of atrial natriuretic factor in Mekk1/- animals, which showed higher mortality and lung/body weight ratio than were seen in controls. Fourteen days after banding, Mekk1/- hearts were dilated, and their left ventricular ejection fraction was low. Pressure overload caused elevated levels of apoptosis and inflammatory lesions in these mice and produced a smaller increase in TGF- $\beta$ and TNF- $\alpha$ expression than occurred in wildtype controls. Thus, MEKK1 appears to be required for pressure overload-induced JNK activation and cytokine upregulation but to be dispensable for pressure overload-induced cardiac hypertrophy. MEKK1 also prevents apoptosis and inflammation, thereby protecting against heart failure and sudden death following cardiac pressure overload.
\end{abstract}

J. Clin. Invest. 110:271-279 (2002). doi:10.1172/JCI200214938.

\section{Introduction}

Mitogen-activated protein kinase (MAPK) pathways play a key role in mediating cell growth and apoptosis (1-3). Accumulating evidence suggests that members of the MAPK family, including extracellular signal-regulated kinase (ERK), c-Jun $\mathrm{NH}_{2}$-terminal kinase (JNK), and p38-MAPKs, play key roles in regulating hypertrophy and apoptosis in cardiac myocytes $(4,5)$. Among the components of the MAPK family, mitogenactivated protein kinase kinase kinase (MEKK1) is a $196-\mathrm{kDa}$ serine-threonine kinase that is activated in response to cytokines and various stresses. MEKK1

Received for publication December 27, 2001, and accepted in revised form June 4, 2002.

Address correspondence to: Junichi Sadoshima, Cardiovascular Research Institute, University of Medicine and Dentistry of New Jersey, New Jersey Medical School, 185 South Orange Avenue, MSB G-609, Newark, New Jersey 07103, USA. Phone: (973) 972-8916; Fax: (973) 972-8919; E-mail: Sadoshju@umdnj.edu. Conflict of interest: No conflict of interest has been declared. Nonstandard abbreviations used: mitogen-activated protein kinase (MAPK); extracellular signal-regulated kinase (ERK); c-Jun $\mathrm{NH}_{2}$-terminal kinase (JNK); mitogen-activated protein kinase kinase kinase (MEKK1); left ventricular (LV); LV end-diastolic diameter (LVEDD); LV end-systolic diameter (LVESD); LV ejection fraction (LVEF); LV weight/body weight (LVW/BW); 4',6-diamidine-2'-phenylindole dihydrochloride (DAPI); atrial natriuretic factor (ANF). preferentially activates the JNK pathway and also influences the activity of the ERK pathway, while it has little or no effect on the p38-MAPK pathway (6-8). The role of MEKK1 and downstream JNKs in cardiac hypertrophy and apoptosis has been studied predominantly in cultured cardiac myocytes in vitro. The results of these studies indicated that JNK is critical in the development of cardiac hypertrophy (9-15). However, an opposing view has been also reported (16). Similarly, conflicting results have been reported regarding the role of MEKK1 and JNKs in cardiac myocyte apoptosis $(4,17-19)$.

Recently, mice deficient in MEKK1 (MEKK1/-- mice) have been generated (20). These mice have defective eyelid closure, a process involving the migration of epithelial cells, but exhibit no apparent abnormality in the heart under base-line conditions. These mice are expected to provide useful information regarding the function of the MEKK1-JNK pathway in the adult mouse heart under stressed conditions in vivo.

We have previously shown that pressure overload causes both cardiac hypertrophy and apoptosis in the mouse heart $(21,22)$. We therefore examined how selective deletion of MEKK1 affects cardiac hypertrophy and apoptosis in adult mice in vivo. In particular, we determined (a) whether JNK activation in response to pressure overload is modified in the MEKK1/- mice 
and (b) whether hypertrophy, survival of animals, and cell death in response to pressure overload are affected in $\mathrm{MEKK} 1^{-/}$mice.

\section{Methods}

$M E K K 1^{-/-}$mice. Generation of MEKK $1^{-/-}$mice has been described previously (20).

Aortic banding. Transverse aortic banding or sham operation was performed in MEKK1-/- mice and littermate $\mathrm{MEKK} 1^{+/+}$mice or nonlittermate $\mathrm{C} 57 \mathrm{BL} / 6$ $\left(\mathrm{MEKK}^{+/+}\right)$mice. Since experiments performed by using littermate $\mathrm{MEKK} 1^{+/+}$mice and nonlittermate C57BL/6 $\left(\mathrm{MEKK}^{+/+}\right)$mice showed indistinguishable results, the data from littermate and nonlittermate MEKK1 $1^{+/}$mice were combined. The method of imposing pressure overload in mice has been described (21, 22). Mice were anesthetized with a mixture of ketamine $(0.065 \mathrm{mg} / \mathrm{g})$, xylazine $(0.013 \mathrm{mg} / \mathrm{g})$, and acepromazine $(0.002 \mathrm{mg} / \mathrm{g})$. Mice were ventilated with a tidal volume of $0.2 \mathrm{ml}$ and a respiratory rate of 110 breaths per minute. The chest was opened at the second intercostal space. Aortic constriction was performed by ligating the transverse thoracic aorta between the innominate artery and left common carotid artery with a 28 -gauge needle using a 7-0 nylon suture. To measure arterial pressure, two high-fidelity catheter tip transducers (1.4F; Millar Instruments Inc., Houston, Texas, USA) were used. One was inserted into the right carotid artery and the other into the left femoral artery, and they were carefully advanced to the ascending aorta and abdominal aorta, respectively, where pressures were measured simultaneously. The pressure gradients between the systolic pressure in the ascending aorta and that in the abdominal aorta were calculated as described (21).

Echocardiography. Mice were anesthetized as described above. Echocardiography was performed using ultrasonography (Apogee CX-200; Interspec Inc., Amber, Pennsylvania, USA) as described previously (23). A dynamically focused 9-MHz annular array transducer was applied from below, using a warmed saline bag as a standoff. M-mode measurements of left ventricular (LV) internal diameter were made from more than three beats and averaged. Measurements of the LV end-diastolic diameter (LVEDD) were taken at the time of the apparent maximal LV diastolic dimension, while measurements of the LV end-systolic diameter (LVESD) were taken at the time of the most anterior systolic excursion of the posterior wall. LV ejection fraction (LVEF) was calculated by the cubed method as follows: $\mathrm{LVEF}=\left[(\mathrm{LVEDD})^{3}-(\mathrm{LVESD})^{3}\right] /(\mathrm{LVEDD})^{3}$.

Evaluation of MAPK activity. Hearts were homogenated with a lysis buffer containing $25 \mathrm{mM}$ Tris, $\mathrm{pH}$ 7.4, $150 \mathrm{mM} \mathrm{NaCl}, 5 \mathrm{mM}$ EDTA, $10 \mathrm{mM}$ sodium pyrophosphate, $10 \mathrm{mM} \beta$-glycerophosphate, $1 \mathrm{mM}$ $\mathrm{Na}_{3} \mathrm{VO}_{4}, 1 \%$ (vol/vol) Triton X-100, $10 \%$ (vol/vol) glycerol, $1 \mathrm{mM}$ dithiothreitol, $1 \mathrm{mM}$ PMSF, and 10 $\mu \mathrm{g} / \mathrm{ml}$ aprotinin. Equal amounts of the heart homogenate $(100 \mu \mathrm{g})$ were separated by SDS-PAGE on
$10 \%$ (wt/vol) gels and transferred onto PVDF membranes. The filters were subjected to immunoblot analyses with anti-phospho-JNK antibody (no. 9255; Cell Signaling Technology Inc., Beverly, Massachusetts, USA), anti-phospho-ERK antibody (no. 9106; Cell Signaling Technology Inc.), or anti-phospho-p38 antibody (no. 9211; Cell Signaling Technology Inc.). Duplicate samples were subjected to immunoblot analyses with anti-JNK1 antibody (sc-474; Santa Cruz Biotechnology Inc., Santa Cruz, California, USA). Some filters were stripped and reprobed with anti-ERK antibody (Zymed Laboratories Inc., South San Francisco, California, USA) or anti-p38 antibody (no. 9212; Cell Signaling Technology Inc.). Blots were quantified by laser scanning densitometry, using NIH Image. The activity of JNK1 was also determined by the immune complex kinase assay using the anti-JNK1 antibody and Glutathione-S-transferase-c-Jun (amino acids 1-79) (Santa Cruz Biotechnology Inc.) as previously described (24).

Histological analyses. The left ventricles, with the septum, were cut into base, mid-portion, and apex, fixed with formalin, embedded in paraffin, and sectioned at $6 \mu \mathrm{m}$ thickness. The sections were incubated in 3\% $\mathrm{H}_{2} \mathrm{O}_{2}$ in PBS to block endogenous peroxidase, and blocked with 5\% BSA in PBS. Primary antibodies were diluted at $1.3-3 \mu \mathrm{g} / \mathrm{ml}$ in PBS, and applied on the sections for 1 hour at $37^{\circ} \mathrm{C}$. After washing, biotinylated secondary antibodies (BD Pharmingen, San Diego, California, USA) were applied for 1 hour, followed by streptavidin/horseradish peroxidase (BD Pharmingen) for 30 minutes at room temperature, and then diaminobenzidine detection system (Vector Laboratories Inc., Burlingame, California, USA) according to the manufacturer's instruction. Nuclear counterstaining was done with hematoxylin. The following primary antibodies were used: rat anti-mouse Mac-3 mAb against marcrophages (BD Pharmingen), rat anti-mouse CD45RA mAb against lymphocytes (BD Pharmingen), and rat anti-mouse $y-6 \mathrm{G}(\mathrm{Gr}-1)$ against neutrophils (BD Pharmingen).

Myocyte cross-sectional area was measured from images captured from silver-stained $1-\mu \mathrm{m}$-thick methacrylate sections as described $(22,23,25)$. Suitable cross sections were defined as having nearly circular capillary profiles and circular-to-oval myocyte sections. No correction for oblique sectioning was made. The

\section{Table 1}

Primers used for RT-PCR analysis

\section{mouse TNF- $\alpha$ (708 bp)}

Sense: ATG-AGC-ACA-GAA-AGC-ATG-ATC

Antisense: TCA-CAG-AGC-AAT-GAC-TCC-AAA

mouse TGF- $\beta 1$ (360 bp)

Sense: ACT-ATG-CTA-AAG-AGG-TCA

Antisense: AGT-GAG-CGC-TGA-ATC-GAA

rat GAPDH (555 bp)

Sense: ATT-GTC-AGC-AAT-GCA-TCC-TG

Antisense: GTA-GGC-CAT-GAG-GTC-CAC-CA 
outline of 100-200 myocytes was traced in each section. MetaMorph image system software (Universal Imaging Corp., Downingtown, Pennsylvania, USA) was used to determine myocyte cross-sectional area $(22,23,25)$.

Evaluation of apoptosis. DNA fragmentation was detected in situ by using TUNEL as described $(22,23)$. Briefly, deparaffinized sections were incubated with proteinase $\mathrm{K}$ and DNA fragments labeled with biotinconjugated dUTP and terminal deoxyribonucleotide transferase, and visualized with FITC-ExtrAvidin (Sigma-Aldrich, St. Louis, Missouri, USA). Nuclear density was determined by manual counting of $4^{\prime}, 6$ diamidine-2'-phenylindole dihydrochloride-stained (DAPI-stained) nuclei in six fields of each animal using the $\times 40$ objective, and the number of TUNEL-positive nuclei was counted by examining the entire section using the same-power objective. Limiting the counting of total nuclei and TUNEL-positive nuclei to areas with a true cross section of myocytes made it possible to selectively count only those nuclei that clearly were within myocytes (25). For some samples, triple staining with propidium iodide (Vector Laboratories Inc.), TUNEL, and anti- $\alpha$-sarcomeric actin antibody (Sigma-Aldrich), and subsequent analyses using confocal microscopy, were performed in order to verify the results obtained with light microscopy. TUNEL-positive cells were also counted in the inflammatory lesions. In this case, the inflammatory area was first identified as described above, and the serial sections were subjected to TUNEL staining.

Northern blot analyses and RT-PCR. Total RNA was isolated from the LV apex part by using Trizol (Life Technologies Inc., Carlsbad, California, USA). Northern blot analyses were performed as described (26). Genomic DNA was removed by incubation with RNase-free DNase. The method of the RT-PCR has been described (27). One microgram of total RNA sample was reversetranscribed in $1 \times$ PCR buffer (Promega Corp., Madison, Wisconsin, USA) in the presence of $5 \mathrm{mM} \mathrm{MgCl}_{2}$, $1 \mathrm{mM}$ dNTPs, $1 \mathrm{U} / \mu \mathrm{l}$ RNase inhibitor, $2.5 \mu \mathrm{M}$ random hexanucleotide primers, and $2.5 \mathrm{U} / \mu \mathrm{l}$ Moloney murine leukemia virus reverse transcriptase. The RT reaction was carried out at $42^{\circ} \mathrm{C}$ for 60 minutes, followed by denaturation at $99^{\circ} \mathrm{C}$ for 5 minutes and cooling at $5^{\circ} \mathrm{C}$ for 5 minutes. PCR was carried out in $1 \times$ PCR buffer containing $2 \mathrm{mM} \mathrm{MgCl}_{2}, 2.5$ units of Taq DNA polymerase, and $0.15 \mu \mathrm{M}$ of the respective primers (Table 1 ). The PCR amplification profile for each of the genes examined involved an initial denaturation step at $95^{\circ} \mathrm{C}$ for 30 seconds to 1 minute. The primer-annealing step was as follows: TGF- $\beta, 63^{\circ} \mathrm{C}$ for 60 seconds; TNF- $\alpha$, $62^{\circ} \mathrm{C}$ for 30 seconds; and GAPDH, $58^{\circ} \mathrm{C}$ for $45 \mathrm{sec}-$ onds. The PCR products were extended for 45 seconds at $72^{\circ} \mathrm{C}$, with a final elongation step at $72^{\circ} \mathrm{C}$ for $7 \mathrm{~min}$ utes. The numbers of PCR cycles were as follows: TGF- $\beta, 30$; TNF- $\alpha, 30$; and GAPDH, 20 . In all cases, PCR of RNA samples was negative in the absence of RT (data not shown). A quantification of gene expression was achieved by the densitometric analysis of the ethidium bromide staining of the PCR products. At fixed PCR cycle numbers, varying input RNA concentrations from 0.1 to $2.5 \mu \mathrm{g}$ resulted in a linear amplification for each gene (not shown).

Statistics. All data are reported as mean \pm SEM. Statistical analyses between groups were done by one-way ANOVA, and when $F$ values were significant at a $95 \%$ confidence limit, differences among group means were evaluated using Fisher's projected least significant difference post-test procedure for group data with $P<0.05$ considered significant.

\section{Results}

JNK activation by pressure overload is abolished in MEKK1-/mice. To examine whether MEKK1 critically mediates JNK activation by pressure overload in the mouse heart, we evaluated activities of JNKs in $\mathrm{MEKK}^{+/+}$ (control) and MEKK1 $1^{-/}$mouse hearts subjected to sham operation or aortic banding for 1,7, and 14 days.

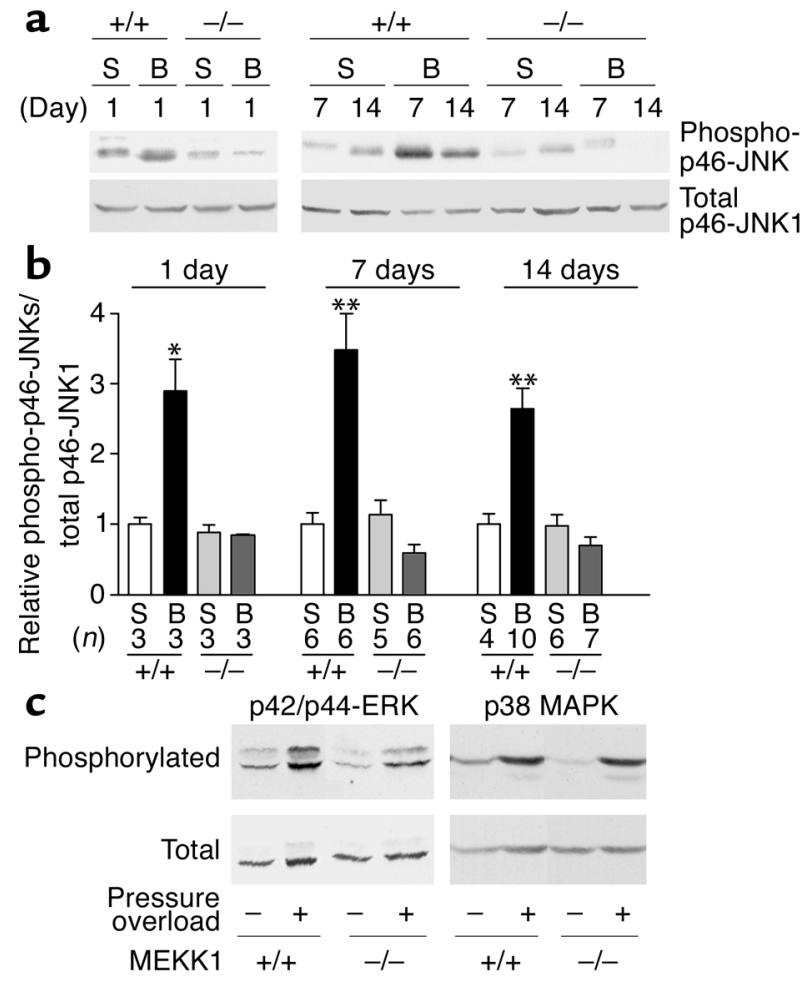

\section{Figure 1}

Activation of JNK by pressure overload is abolished, while that of ERKs and p38-MAPKs is preserved, in MEKK1/- mice. Aortic banding (B) or sham operation (S) was performed in $\mathrm{MEKK} 1^{+/+}$or MEKK $1^{-1-}$ mice. The animals were sacrificed at 1,7 , or 14 days. (a and $\mathbf{b}$ ) The kinase activity of $\mathrm{p} 46-\mathrm{JNK}$ was determined by anti-phosphospecific JNK antibody. Duplicate samples were subjected to immunoblot analyses with anti-JNK1 antibody. $\ln \mathbf{b}$, the mean of the phospho-p46-JNKs/total JNK1 in control mice with sham operation is expressed as 1 in each group. ${ }^{*} P<0.05,{ }^{*} P<0.001 \mathrm{vs}$. control sham-operated in each group. (c) Animals were sacrificed at 7 days. The activity of ERKs and p38-MAPKs was evaluated by immunoblotting with anti-phosphospecific antibody. The filters were reprobed with anti-non-phosphospecific antibody after stripping. The results were representative of five samples per group. 

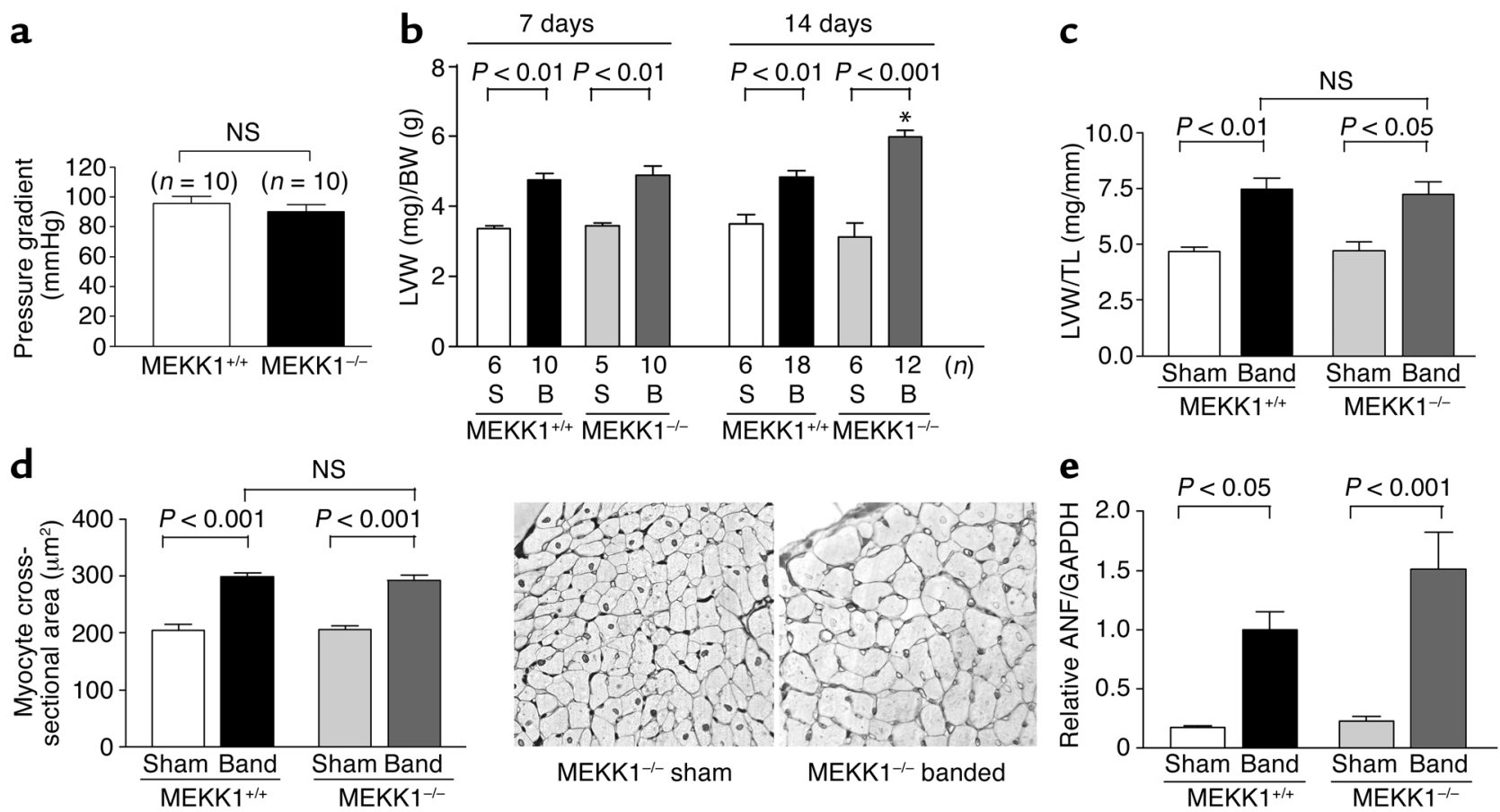

\section{Figure 2}

Cardiac hypertrophy by pressure overload is preserved in MEKK1/- mice. Transverse aortic banding or sham operation was applied in either control or MEKK1/- mice. (a) Pressure gradient across the constriction at the transverse aorta was measured at 7 days. (b) LV weight (LVW) (mg)/body weight (BW) (g) was obtained at 7 and 14 days. ${ }^{*} P<0.001 \mathrm{vs.} \mathrm{MEKK1+/+}$ mice with 14 days banding. (c) LVW $(\mathrm{mg}) /$ tibial length $(\mathrm{TL})(\mathrm{mm})$ was obtained at 7 days. $n=5-10$. (d) Cardiac myocyte cross-sectional area was obtained at 7 days. $n=5$ for each group. The right panel shows the light micrographs of silver-stained $1-\mu \mathrm{m}$ sections of LV myocardium from sham-operated and banded MEKK1/- mice. (e) mRNA expression of atrial natriuretic factor (ANF) at 7 days was evaluated by Northern blot analyses. Densitometric analyses were performed. The mean of ANF/GAPDH in control mice subjected to 1 week of banding was expressed as 1 . $n=5$ for each group. NS, not significant.

Immunoblot analyses with anti-phospho-JNK antibody indicated 2.9-, 3.5-, and 2.6-fold increases in phospho-p46-JNK in response to pressure overload for 1,7 , and 14 days, respectively, compared with sham operation in control mice (Figure 1, a and b). Interestingly, pressure overload for 1,7 , or 14 days failed to increase kinase activities of $\mathrm{p} 46-\mathrm{JNK}$ in MEKK1-/- mice compared with those subjected to sham operation (Figure 1, a and b). Longer exposures of the immunoblot showed that there were no significant changes in p54-JNK activity after banding compared with sham operation in control or MEKK1 $1^{-/}$mice (not shown). Immunoblot analyses of the duplicate samples with anti-JNK1 antibody showed that comparable amounts of the samples were quantitated in these experiments. Immune complex kinase assays using anti-JNK1 antibody confirmed that the activity on JNK1 was significantly increased by aortic banding for
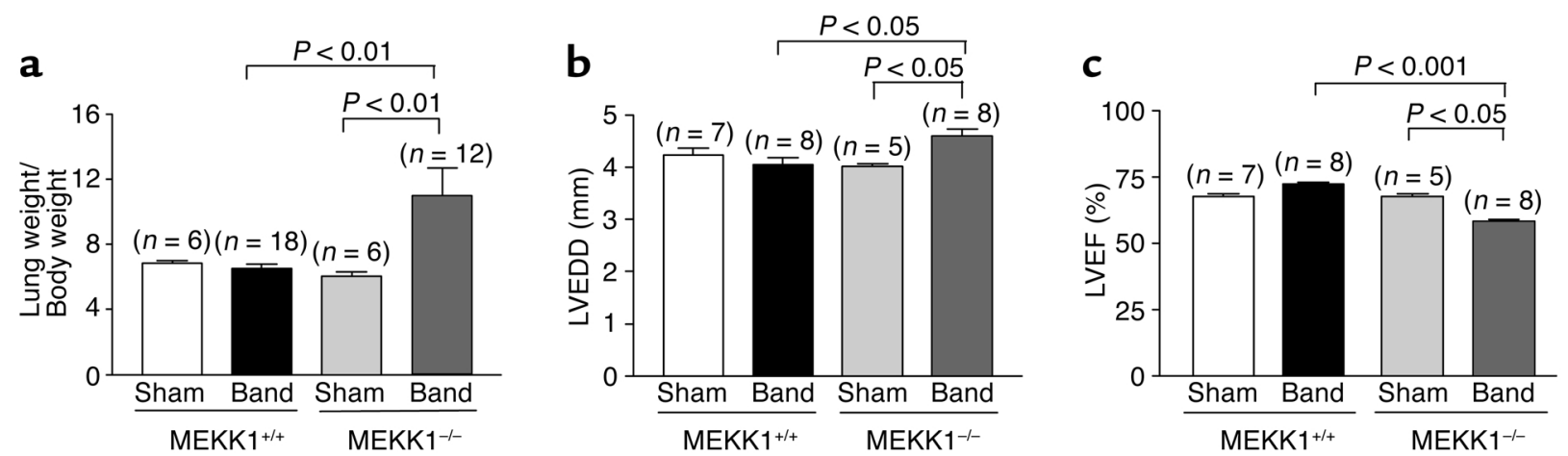

Figure 3

Pressure overload in MEKK1/- mice caused increases in the lung/body weight and LV end-diastolic dimension and decreases in LVEF. Aortic banding or sham operation was applied in control or MEKK1/- mice. (a) The lung weight and the body weight were determined at 14 days. ( $b$ and $\mathbf{c}$ ) Echocardiographic measurements were performed at 14 days. LVEDD and LVEF were determined. 
a
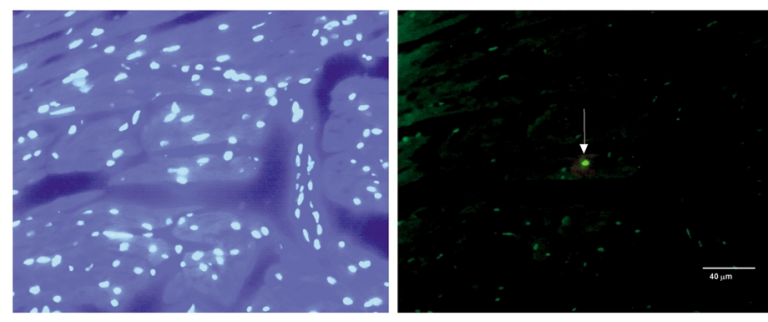

b

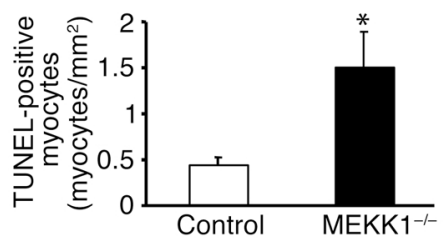

C

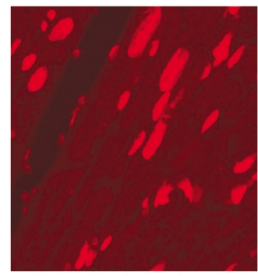

Propidium iodide + $\alpha$-sarcomeric actin

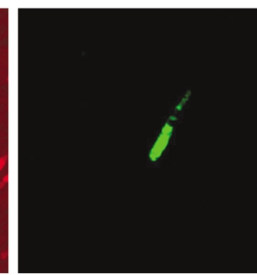

TUNEL

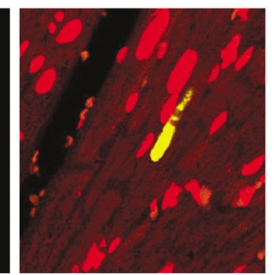

Overlay
7 days in control mice but not in MEKK1/- mice, compared with sham operation in each group (data not shown). These results suggest that MEKK1 plays an essential role in mediating $\mathrm{p} 46-\mathrm{JNK}$ activation by pressure overload in mice.

We examined how other members of the MAPK family are affected by pressure overload in our model. In control mice, pressure overload for 7 days caused significant increases in both phospho-ERKs and phospho-p38-MAPK compared with sham-operated mice (Figure 1c). In MEKK1 $1^{--}$mice, the level of phosphoERKs and phospho-p38-MAPK in sham-operated mice was similar to that in control mice, and pressure overload caused comparable levels of increases in both phospho-ERKs and phospho-p38-MAPK compared with those in control mice (Figure 1c).

Pressure overload causes significant levels of cardiac bypertrophy in MEKK1-/- mice. We examined whether pressure overload-induced cardiac hypertrophy is affected in MEKK1-- mice. Neither the body weight nor the heart weight was significantly different between control and

\section{Figure 5}

Pressure overload causes more inflammatory lesions, predominantly consisting of macrophages, as well as apoptosis of the inflammatory cells in the left ventricle in MEKK1 $1^{-/-}$mice. Hematoxylin-andeosin staining reveals an increased number of inflammatory lesions in $\mathrm{MEKK} 1^{-1-}$ mice (b) compared with control mice (a). Bar $=150$ $\mu \mathrm{m}$.(c) The inflammatory lesions in LV myocardium were measured by image analysis software. There was an increase in inflammatory lesions in MEKK1 $1^{-/-}$mice compared with control mice after 7 days of pressure overload. ${ }^{*} P<0.05 ; n=5$. (d) Macrophages, lymphocytes, and neutrophils in the LV myocardium from MEKK1-/- mice subjected to pressure overload were stained as described in Methods. The inflammatory cells primarily consisted of macrophages.

\section{Figure 4}

Pressure overload causes more TUNEL-positive cells in the left ventricle in MEKK1/- mice. ( $\mathbf{a}$ and $\mathbf{b}$ ) DAPI staining (left) and TUNEL staining (right) of the LV myocardium 7 days after aortic banding in a MEKK1 $1 /$ mouse. The white arrow indicates a TUNEL-positive nucleus. Bar $=40 \mu \mathrm{m}$. (b) TUNEL-positive myocytes in LV myocardium were counted in control and MEKK $1^{-/-}$mice subjected to aortic banding for 7 days and expressed as the number per $\mathrm{mm}^{2}$. The number of TUNEL-positive myocytes was significantly higher in MEKK $1{ }^{-/-}$mice than in the control mice. ${ }^{*} P<0.05 ; n=5$. (c) Images of the confocal microscopic analyses showing nuclear fragmentation in a MEKK $1^{-/-}$mouse banded for 7 days. Triple staining (propidium iodide, TUNEL, and anti- $\alpha$-sarcomeric actin antibody) was performed. Staining for propidium iodide and anti- $\alpha$-sarcomeric actin antibody is shown by red, and that for TUNEL by green. In the overlay image, a nucleus stained by both TUNEL and propidium iodide is shown by yellow.

MEKK1 $1^{--}$mice before banding (data not shown). This suggests that MEKK1 does not play an essential role in heart development, in contrast to MEKK3, which plays a critical role in cardiovascular development (28). After 7 and 14 days of banding, the induced pressure gradient was similar in MEKK1 $1^{-/}$and control mice (Figure $2 \mathrm{a}$ and data not shown). As expected, in control mice, aortic banding for either 7 or 14 days increased LV weight/body weight (LVW/BW) significantly. In MEKK1/- mice, aortic banding caused significant increases in $\mathrm{LVW} / \mathrm{BW}$ at 7 and 14 days (Figure $2 \mathrm{~b}$ ). The value of LVW/BW at 14 days in MEKK1/- mice was even higher than that in control mice. Cardiac hypertrophy by aortic banding in MEKK $1^{-/}$mice was also confirmed by LVW/tibial length, another indicator of cardiac hypertrophy, which was measured at 7 days (Figure 2c). Cardiac myocyte cross-sectional area was significantly increased after banding in both control and MEKK $1^{-/-}$mice, and there was
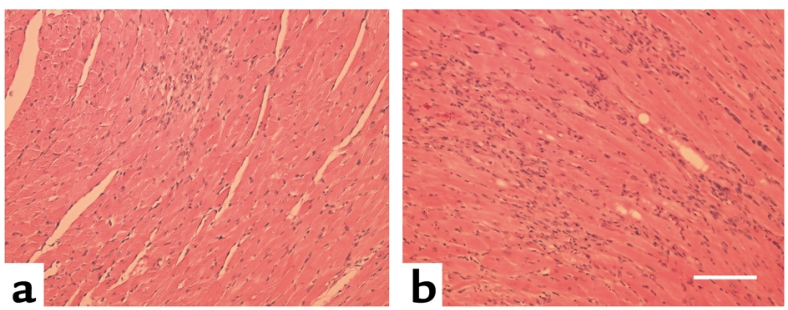

C

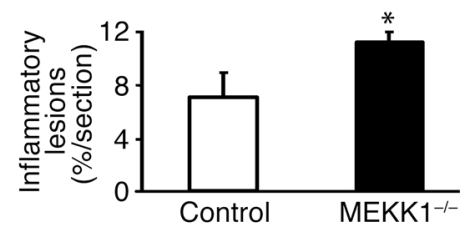

d

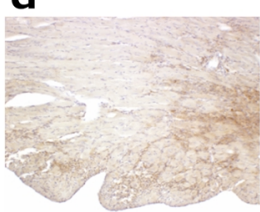

Macrophages

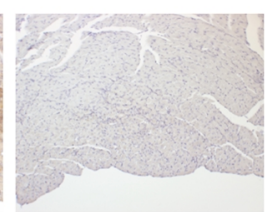

Lymphocytes

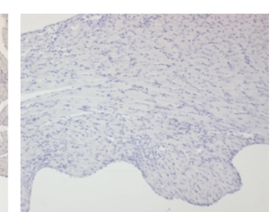

Neutrophils 

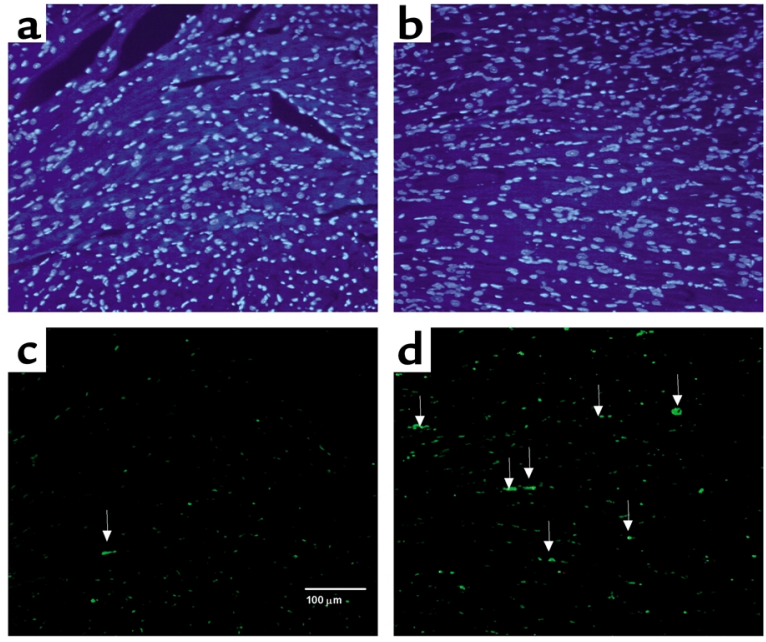

e

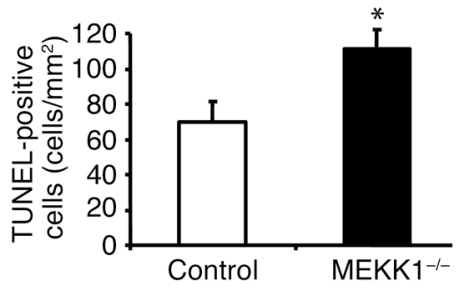

no significant difference in myocyte cross-sectional area between control and MEKK1/- mice after 7 days of aortic banding (Figure 2d).

mRNA expression of atrial natriuretic factor (ANF), a fetal-type gene frequently upregulated in many types of cardiac hypertrophy (26), was determined by Northern blot analyses. ANF expression was upregulated by aortic banding for 7 days in both control and MEKK1 $1^{--}$mice. Although ANF expression after banding tended to be higher in MEKK $1^{-/-}$mice, there was no statistically significant difference in the level of ANF expression between these two groups (Figure 2e). Taken together, cardiac hypertrophy develops in response to pressure overload in MEKK1-/- mice.

Pressure overload causes increased mortality and congestive heart failure in MEKK1 $1^{-1-}$ mice. Although pressure overload caused significant levels of cardiac hypertrophy in both control and MEKK1 $1^{-/}$mice, MEKK1 ${ }^{-/}$mice

\section{Figure 6}

Pressure overload causes more apoptosis in the inflammatory lesions of MEKK $1^{-/-}$mice than in those of MEKK $1^{+/+}$mice. MEKK $1^{+/+}$(control) and MEKK1 $1^{-/-}$mice were subjected to aortic banding for 7 days. DAPI staining (a and $\mathbf{b}$ ) and TUNEL staining ( $\mathbf{c}$ and $\mathbf{d}$ ) in LV myocardium 7 days after aortic banding. White arrows indicate TUNEL-positive myocyte nuclei in a control mouse ( $\mathbf{a}$ and $\mathbf{c}$ ) and in a MEKK $1^{-/}$mouse (b and d). Bar $=100 \mu \mathrm{m}$. (e) TUNEL-positive cells in the inflammatory lesions in LV myocardium. More TUNEL-positive cells were observed in the inflammatory lesions of MEKK $1^{-1-}$ mice than in those of control mice. ${ }^{*} P<0.05$.

exhibited higher mortality during 14 days of aortic banding. While none of 18 control mice died during 2 weeks after banding, 4 out of $16 \mathrm{MEKK} 1^{-/-}$mice died between days 7 and 14. In control mice, the lung/body weight of the 14-day aortic banding group did not significantly differ from that of the sham group. By contrast, in MEKK1/- mice, the lung/body weight in the 14-day aortic banding group was significantly higher than that in the sham group (Figure 3a). The lung/body weight in MEKK1 $1^{-/}$mice subjected to aortic banding may be underestimated, because we could not obtain the lung/body weight from the animals that died before 14 days of aortic banding.

LV dimension and function were evaluated echocardiographically. The LVEDD and LVEF after 7 days of banding in control or MEKK1 $1^{-/}$mice were not different from those in each sham group (data not shown). LVEDD and LVEF in control mice were maintained at 14 days after banding (Figure 3, b and c). By contrast, LVEDD was significantly increased while LVEF was significantly reduced $(P<0.05)$ in MEKK1 $1^{-/-}$mice after 14 days of banding compared with the sham group (Figure $3, b$ and $c$ ). These results suggest that MEKK1-mice do not tolerate pressure overload as well as wildtype mice do, and they are consistent with the finding that the lung/body weight was increased in MEKK1-mice. The impaired cardiac function and pulmonary congestion indicate that MEKK1/-- mice actually develop heart failure in response to pressure overload.

Pressure overload causes more cell death and inflammatory regions in $\mathrm{MEKK1^{-/- }}$ mice. There was no significant difference in basal levels of TUNEL-positive cells

\footnotetext{
Figure 7

Upregulation of TNF- $\alpha$ and TGF- $\beta$ expression by pressure overload is impaired in MEKK $1^{-/-}$mice. $\mathrm{MEKK}^{+/+}$(control) and MEKK1 ${ }^{-/-}$mice were subjected to either aortic banding or sham operation. After 7 days of pressure overload, animals were sacrificed and total RNA was isolated from the apex. mRNA expression of TNF- $\alpha$ and TGF- $\beta$ was determined by RT-PCR. Expression of GAPDH was determined as an internal control. Representative ethidium bromide staining of the products of RT-PCR is shown. $n=5$.
}
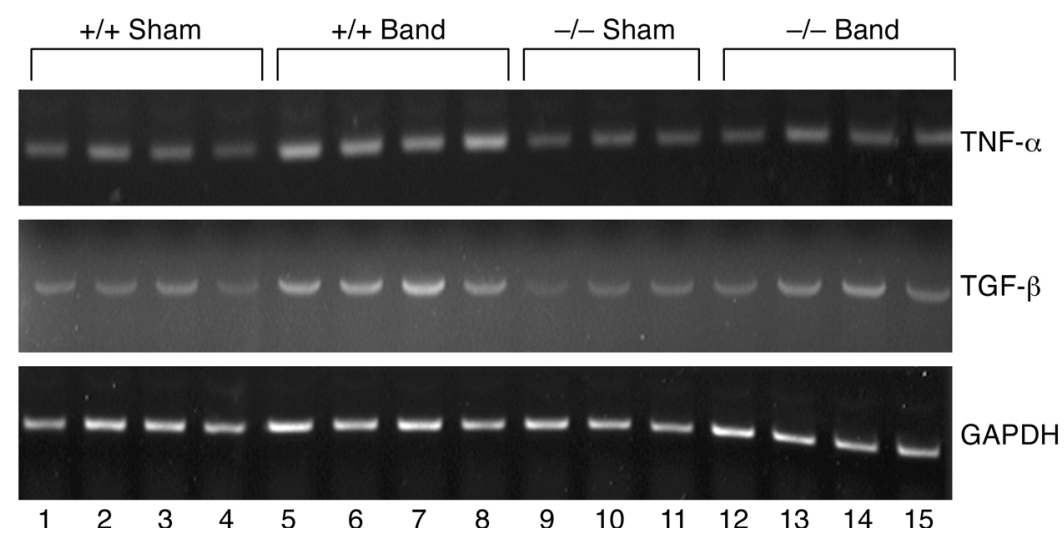
between control and MEKK1/- mice. Surprisingly, 7 days after aortic banding, significantly more TUNELpositive myocytes were observed in the LV myocardium obtained from $\mathrm{MEKK}^{-/}$mice $(1.50 \pm 0.39$ myocytes $/ \mathrm{mm}^{2}$ ) than in that from control mice $\left(0.44 \pm 0.08\right.$ myocytes $\left./ \mathrm{mm}^{2}, P=0.02\right)$ (Figure 4 , a and b). The increased level of cardiac myocyte apoptosis was confirmed by triple staining (propidium iodide, TUNEL, and anti- $\alpha$-sarcomeric actin antibody) and subsequent confocal microscopic analyses (TUNELpositive nuclei per 30 fields, MEKK1/- mice $[n=4]$ vs. control mice $[n=5]: 5 \pm 1.32$ vs. $2 \pm 0.37, P<0.05$ ) (Figure 4c). Aortic banding caused multifocal inflammatory lesions in both control and MEKK1/- mice. Interestingly, more inflammatory lesions were found in MEKK $1^{-/}$mice $(11 \% \pm 1 \%$ per section $)$ than in control mice $(7 \% \pm 2 \%$ per section) after banding (Figure 5, a, b, and c). Histochemical analyses of the heart sections indicated that the predominant cell type in the inflammatory lesions was macrophages (Figure 5d). Interestingly, more TUNEL-positive cells were observed also in the inflammatory lesions of the left ventricles obtained from MEKK1 $1^{-/}$mice $\left(111.1 \pm 11.1\right.$ cells $\left./ \mathrm{mm}^{2}\right)$ than in those from control mice $\left(69.7 \pm 11.9\right.$ cells $\left./ \mathrm{mm}^{2}, P=0.04\right)$ (Figure 6).

Pressure overload-induced upregulation of cytokine expression is impaired in $M E K K 1^{-1-}$ mice. It has been shown that expression of cytokines is significantly affected by targeted deletion of MEKK1 in embryonic stem cell-derived cardiac myocytes (17). We therefore examined how expression of two representative cytokines, namely TGF- $\beta$ and TNF- $\alpha$, whose expression has been shown to be altered during load-induced cardiac hypertrophy and heart failure (29-31), are affected by pressure overload in MEKK1/- mice. The results of RT-PCR analyses indicated that pressure overload for 7 days increased mRNA expression of TGF- $\beta$ and TNF- $\alpha$ in control mice. Although basal expression of TGF- $\beta$ and TNF- $\alpha$ was similar between control and MEKK1-/mice subjected to sham operation, aortic banding caused a smaller increase in TGF- $\beta$ and TNF- $\alpha$ expression in $\mathrm{MEKK}^{-/-}$mice than in control mice (1.3- and 1.2-fold in MEKK1-- mice vs. 1.6- and 2.2-fold in control mice) (Figure 7).

\section{Discussion}

Pressure overload-induced cardiac hypertrophy occurs even without activation of the MEKK1-JNK pathway. A major conclusion in this report is that cardiac hypertrophy by pressure overload occurs even in the absence of JNK activation in MEKK1/- mice. Although activation of JNK has been suggested to play an important role in pressure overload-induced cardiac hypertrophy (32), parallel signaling mechanisms seem sufficient to compensate and permit cardiac hypertrophy to develop normally in response to pressure overload. Our results are distinct from those of a recent study showing that cardiac hypertrophy and LV dysfunction in transgenic mice with cardiac-specific overexpression of $\mathrm{G} \alpha \mathrm{q}$ are significantly attenuated by crossing the animals with MEKK1 $1^{-/}$mice (33). This suggests that the signaling mechanisms of cardiac hypertrophy and subsequent cardiac decompensation by pressure overload and Goq are not identical despite the fact that secretion and production of agonists for Goq-coupled receptors play an important role in mediating pressure overload-induced cardiac hypertrophy (34). It has been shown that JNK activation by pressure overload is significantly, if not completely, inhibited by the Goq carboxyl-terminus peptide (Goq inhibitor) (35). Since mechanical stress as well as multiple autocrine/paracrine factors activate multiple parallel signaling mechanisms (reviewed in refs. 5, 34), we speculate that suppressing only one signaling pathway, namely the MEKK1-JNK pathway, may not be sufficient to suppress cardiac hypertrophy by pressure overload.

MEKK1 may play an essential role in maintaining cardiac function in response to pressure overload. In the present study, the animals subjected to aortic banding developed cardiac hypertrophy without overt signs of congestive heart failure until 7 days in both control and MEKK1/-- groups. Interestingly, more MEKK1/-- mice than $\mathrm{MEKK} 1^{+/+}$died after 7 days of banding. The lung/body weight, an index of heart failure, in MEKK1/- mice after 14 days of banding was significantly higher than that in control mice. The left ventricle was dilated and the LVEF was not maintained as well in MEKK $1^{-/-}$mice in response to pressure overload at 14 days. These results suggest that MEKK1-/mice undergo transition from compensated to decompensated stages during the second week of banding and develop congestive heart failure. Although the exact cellular mechanism for MEKK1 $1^{-/}$to develop heart failure remains to be elucidated, interestingly, MEKK1/- mice subjected to pressure overload exhibited more severe macrophage infiltration and more TUNEL-positive cells in the LV myocardium than did wild-type animals even before the animals exhibited signs of congestive heart failure. Since both inflammation (36) and cell death, including apoptosis (37), are intimately involved in development of congestive heart failure, we speculate that enhanced inflammatory response and cardiac myocyte apoptosis may facilitate transition from compensated LV hypertrophy to a decompensated one in MEKK $1^{-/-}$mice. The fact that both inflammation and cell death are enhanced by pressure overload but not by sham operation in MEKK1 $1^{-/-}$mice indicates that MEKK1 and JNK activated by pressure overload should normally prevent pressure overload-induced inflammation and cell death in control hearts.

Pressure overload causes more cell death in MEKK1-/mice. It has been shown that overexpression of constitutively active MEKK1 in cardiac myocytes promotes apoptosis, which is presumably mediated by JNK (38). JNKs play a critical role in mediating cardiac myocyte apoptosis by ischemia/reperfusion (19). In this regard, 
the result that MEKK1/- mice showed more TUNELpositive cells after banding was surprising, considering the fact that activation of JNKs by pressure overload is impaired in this animal model. Our results are consistent with the recent observation that cardiac myocytes derived from MEKK1 $1^{-/-}$embryonic stem cells showed enhanced apoptosis in response to $\mathrm{H}_{2} \mathrm{O}_{2}$ stimulation in vitro (17). Furthermore, activation of JNK protects cardiac myocytes from apoptosis by nitric oxide (18). Thus, the role of the MEKK1-JNK pathway in cell survival and apoptosis is cell type- as well as stimulus-specific. We speculate that activation of the MEKK1-JNK pathway by pressure overload leads to stimulation of the cytoprotective mechanisms in the heart.

Upregulation of TNF- $\alpha$ and TGF- $\beta$ by pressure overload is impaired in MEKK1-/ mice. TNF- $\alpha$ and TGF- $\beta$ are two representative cytokines, which are upregulated by pressure overload and play an important role in cardiac hypertrophy $(29,30)$ and heart failure (31). Interestingly, upregulation of these cytokines by pressure overload was impaired in MEKK1/- mice, suggesting that the MEKK1-JNK pathway plays a critical role in upregulation of TNF- $\alpha$ and TGF- $\beta$ during pressure overload-induced cardiac hypertrophy. This result also suggests that pressure overload-induced cardiac hypertrophy occurs even in the absence of upregulation of TNF- $\alpha$ or TGF- $\beta$. Interestingly, decompensation in response to pressure overload occurred in the absence of TNF- $\alpha$ and TGF- $\beta$, which does not support the role of these cytokines in the pathogenesis of heart failure. It has been shown that TNF- $\alpha$ expression in response to $\mathrm{H}_{2} \mathrm{O}_{2}$ was enhanced in cardiac myocytes derived from MEKK $1^{-/-}$mouse embryonic stem cells, causing enhanced apoptosis (17). It is likely that the signaling mechanism regulating TNF- $\alpha$ expression in vivo is different from that in embryonic stem cell-derived cardiac myocytes in vitro. Although TNF- $\alpha$ has been reported to promote apoptosis in some cell types, including cardiac myocytes $(39,40)$, recent studies have indicated that increased expression of TNF- $\alpha$ in myocardium does not stimulate cardiac myocyte apoptosis $(41,42)$. Furthermore, it has recently been suggested that TNF- $\alpha$ rather protects cardiac myocytes from apoptosis through activation of NF- $\mathrm{KB}$ and subsequent expression of other cytokines, such as IL-6. Enhanced levels of cardiac myocyte apoptosis were observed in mice deficient in both type 1 and type 2 TNF receptors and subjected to myocardial ischemia (43). Similarly, TGF- $\beta 1$ has been shown to protect adult cardiac myocytes from apoptosis by hypoxia/reoxygenation (44), and enhanced levels of cardiac myocyte apoptosis were observed in mice deficient in TGF- $\beta 2$ (45). These results suggest that the MEKK1-JNK signaling pathway mediates upregulation of cardioprotective cytokines in response to pressure overload, thereby preventing transition from compensatory to decompensated stages of cardiac hypertrophy and playing a protective role during pressure overload.

\section{Acknowledgments}

This work was in part supported by grants from the NIH (HL-59139, HL-33107, HL-33065, HL-65182, HL-65183, AG-14121, HL-69020, HL-67724, HL-67727, GM-30324, DK37871) and the American Heart Association National Center (9950673N).

1. Widmann, C., Gibson, S., Jarpe, M.B., and Johnson, G.L. 1999. Mitogenactivated protein kinase: conservation of a three-kinase module from yeast to human. Physiol. Rev. 79:143-180.

2. Pearson, G., et al. 2001. Mitogen-activated protein (MAP) kinase pathways: regulation and physiological functions. Endocr. Rev. 22:153-183.

3. Davis, R.J. 2000. Signal transduction by the JNK group of MAP kinases. Cell. 103:239-252.

4. Sugden, P.H., and Clerk, A. 1998. "Stress-responsive" mitogen-activated protein kinases (c-Jun $\mathrm{N}$-terminal kinases and p38 mitogen-activated protein kinases) in the myocardium. Circ. Res. 83:345-352.

5. Molkentin, J.D. and Dorn, I.G., II. 2001. Cytoplasmic signaling pathways that regulate cardiac hypertrophy. Annu. Rev. Physiol. 63:391-426.

6. Lange-Carter, C.A., Pleiman, C.M., Gardner, A.M., Blumer, K.J., and Johnson, G.L. 1993. A divergence in the MAP kinase regulatory network defined by MEK kinase and Raf. Science. 260:315-319.

7. Yujiri, T., Sather, S., Fanger, G.R., and Johnson, G.L. 1998. Role of MEKK1 in cell survival and activation of JNK and ERK pathways defined by targeted gene disruption. Science. 282:1911-1914.

8. Yujiri, T., et al. 1999. MEK kinase 1 (MEKK1) transduces c-Jun NH2-terminal kinase activation in response to changes in the microtubule cytoskeleton. J. Biol. Chem. 274:12605-12610.

9. Thorburn, J., Xu, S., and Thorburn, A. 1997. MAP kinase- and Rhodependent signals interact to regulate gene expression but not actin morphology in cardiac muscle cells. EMBOJ. 16:1888-1900.

10. Bogoyevitch, M.A., et al. 1996. Stimulation of the stress-activated mitogen-activated protein kinase subfamilies in perfused heart. p38/RK mitogen-activated protein kinases and c-Jun $\mathrm{N}$-terminal kinases are activated by ischemia/reperfusion. Circ. Res. 79:162-173.

11. Ramirez, M.T., et al. 1997. The MEKK-JNK pathway is stimulated by alpha1-adrenergic receptor and ras activation and is associated with in vitro and in vivo cardiac hypertrophy. J. Biol. Chem. 272:14057-14061.

12. McDonough, P.M., Hanford, D.S., Sprenkle, A.B., Mellon, N.R., and Glembotski, C.C. 1997. Collaborative roles for c-Jun N-terminal kinase, c-Jun, serum response factor, and $\mathrm{Sp} 1$ in calcium-regulated myocardial gene expression. J. Biol. Chem. 272:24046-24053.

13. Wang, Y., et al. 1998. Cardiac hypertrophy induced by mitogen-activated protein kinase kinase 7, a specific activator for c-Jun NH2-terminal kinase in ventricular muscle cells. J. Biol. Chem. 273:5423-5426.

14. Choukroun, G., et al. 1999. Regulation of cardiac hypertrophy in vivo by the stress-activated protein kinases/c-Jun $\mathrm{NH}(2)$-terminal kinases. J. Clin. Invest. 104:391-398.

15. Choukroun, G., et al. 1998. Role of the stress-activated protein kinases in endothelin-induced cardiomyocyte hypertrophy. J. Clin. Invest. 102:1311-1320.

16. Nemoto, S., Sheng, Z., and Lin, A. 1998. Opposing effects of Jun kinase and p38 mitogen-activated protein kinases on cardiomyocyte hypertrophy. Mol. Cell. Biol. 18:3518-3526.

17. Minamino, T., et al. 1999. MEKK1 suppresses oxidative stress-induced apoptosis of embryonic stem cell-derived cardiac myocytes. Proc. Natl. Acad. Sci. USA. 96:15127-15132.

18. Andreka, P., et al. 2001. Cytoprotection by Jun kinase during nitric oxideinduced cardiac myocyte apoptosis. Circ. Res. 88:305-312.

19. Hreniuk, D., et al. 2001. Inhibition of c-Jun $\mathrm{N}$-terminal kinase 1, but not c-Jun N-terminal kinase 2, suppresses apoptosis induced by ischemia/reoxygenation in rat cardiac myocytes. Mol. Pharmacol. 59:867-874.

20. Yujiri, T., et al. 2000. MEK kinase 1 gene disruption alters cell migration and c-Jun NH2-terminal kinase regulation but does not cause a measurable defect in NF-kappa B activation. Proc. Natl. Acad. Sci. USA. 97:7272-7277.

21. Meguro, T., et al. 1999. Cyclosporine attenuates pressure-overload hypertrophy in mice while enhancing susceptibility to decompensation and heart failure. Circ. Res. 84:735-740.

22. Yang, G., et al. 2000. Cyclosporine reduces left ventricular mass with chronic aortic banding in mice, which could be due to apoptosis and fibrosis. J. Mol. Cell. Cardiol. 33:1505-1514.

23. Asai, K., et al. 1999. Beta-adrenergic receptor blockade arrests myocyte damage and preserves cardiac function in the transgenic $G($ salpha) mouse. J. Clin. Invest. 104:551-558.

24. Aoki, H., Richmond, M., Izumo, S., and Sadoshima, J. 2000. Specific role of the extracellular signal-regulated kinase pathway in angiotensin IIinduced cardiac hypertrophy in vitro. Biochem. J. 347:275-284. 
25. Geng, Y.J., et al. 1999. Apoptosis of cardiac myocytes in Gsalpha transgenic mice. Circ. Res. 84:34-42.

26. Sadoshima, J., Jahn, L., Takahashi, T., Kulik, T.J., and Izumo, S. 1992. Molecular characterization of the stretch-induced adaptation of cultured cardiac cells. An in vitro model of load-induced cardiac hypertrophy. J. Biol. Chem. 267:10551-10560.

27. Malhotra, R., Sadoshima, J., Brosius, F.C., III, and Izumo, S. 1999. Mechanical stretch and angiotensin II differentially upregulate the renin-angiotensin system in cardiac myocytes in vitro. Circ. Res. 85:137-146.

28. Yang, J., et al. 2000. Mekk3 is essential for early embryonic cardiovascular development. Nat. Genet. 24:309-313.

29. Kapadia, S.R., et al. 1997. Hemodynamic regulation of tumor necrosis factor-alpha gene and protein expression in adult feline myocardium. Circ. Res. 81:187-195.

30. Takahashi, N., et al. 1994. Hypertrophic stimuli induce transforming growth factor-beta 1 expression in rat ventricular myocytes. J. Clin. Invest. 94:1470-1476.

31. Deswal, A., et al. 2001. Cytokines and cytokine receptors in advanced heart failure: an analysis of the cytokine database from the vesnarinone trial (VEST). Circulation. 103:2055-2059.

32. Fischer, T.A., et al. 2001. Activation of cardiac c-Jun NH(2)-terminal kinases and $\mathrm{p} 38$-mitogen-activated protein kinases with abrupt changes in hemodynamic load. Hypertension. 37:1222-1228.

33. Minamino, T., et al. 2002. MEKK1 is essential for cardiac hypertrophy and dysfunction induced by Gq. Proc. Natl. Acad. Sci. USA. 99:3866-3871.

34. Sadoshima, J., and Izumo, S. 1997. The cellular and molecular response of cardiac myocytes to mechanical stress. Annu. Rev. Physiol. 59:551-571.

35. Esposito, G., et al. 2001. Cardiac overexpression of a G(q) inhibitor blocks induction of extracellular signal-regulated kinase and c-Jun $\mathrm{NH}(2)$-terminal kinase activity in in vivo pressure overload. Circulation. 103:1453-1458.
36. Matsumori, A., Yamada, T., Suzuki, H., Matoba, Y., and Sasayama, S 1994. Increased circulating cytokines in patients with myocarditis and cardiomyopathy. Br. Heart J. 72:561-566.

37. Kang, P.M., and Izumo, S. 2000. Apoptosis and heart failure: a critical review of the literature. Circ. Res. 86:1107-1113.

38. Zechner, D., Thuerauf, D.J., Hanford, D.S., McDonough, P.M., and Glembotski, C.C. 1997. A role for the p38 mitogen-activated protein kinase pathway in myocardial cell growth, sarcomeric organization, and cardiac-specific gene expression. J. Cell Biol. 139:115-127.

39. Hsu, H., Xiong, J., and Goeddel, D.V. 1995. The TNF receptor 1-associated protein TRADD signals cell death and NF-kappa B activation. Cell. 81:495-504.

40. Krown, K.A., et al. 1996. Tumor necrosis factor alpha-induced apoptosis in cardiac myocytes. Involvement of the sphingolipid signaling cascade in cardiac cell death. J. Clin. Invest. 98:2854-2865.

41. Kubota, T., et al. 2001. Overexpression of tumor necrosis factor-alpha activates both anti- and pro-apoptotic pathways in the myocardium. J. Mol. Cell. Cardiol. 33:1331-1344.

42. Nelson, D.P., et al. 2000. Proinflammatory consequences of transgenic fas ligand expression in the heart. J. Clin. Invest. 105:1199-1208.

43. Kurrelmeyer, K.M., et al. 2000. Endogenous tumor necrosis factor protects the adult cardiac myocyte against ischemic-induced apoptosis in a murine model of acute myocardial infarction. Proc. Natl. Acad. Sci. USA. 97:5456-5461.

44. Yang, B.C., Zander, D.S., and Mehta, J.L. 1999. Hypoxia-reoxygenationinduced apoptosis in cultured adult rat myocytes and the protective effect of platelets and transforming growth factor-beta(1). J. Pharmacol. Exp. Ther. 291:733-738.

45. Bartram, U., et al. 2001. Double-outlet right ventricle and overriding tricuspid valve reflect disturbances of looping, myocardialization, endocardial cushion differentiation, and apoptosis in TGF-beta(2)-knockout mice. Circulation. 103:2745-2752. 